\title{
THE COMPILED CATALOGUE OF PHOTOELECTRIC UBVR STELLAR MAGNITUDES IN THE TYCHO2 SYSTEM
}

\author{
E.Relke $^{1}$, Yu.I.Protsyuk ${ }^{2}$, V.M.Andruk ${ }^{3}$ \\ ${ }^{1}$ Walter-Hohmann-Observatory, 159 Wallneyer St., 45133 Essen, \\ Germany,helena_relke@yahoo.com \\ ${ }^{2}$ Research Institute: Nikolaev Astronomical Observatory, \\ 1 Observatornaya St., 54030, Mykolaiv, Ukraine,yuri@nao.nikolaev.ua \\ ${ }^{3}$ Main Astronomical Observatory of National Academy of Sciences, \\ 27 Akad. Zabolotnogo St., 03680, Kyiv, Ukraine, andruk@mao.kiev.ua
}

\begin{abstract}
In order to calibrate the images of astronomical photographic plates from the archive of UkrVO was created the compiled catalogue of photoelectric UBVR stellar magnitudes. It is based on: the Kornilov catalogue of 13586 WBVR stellar magnitudes (Kornilov at al., 1991), the Mermilliod catalogue of $68540 \mathrm{UBV}$ stellar magnitudes (Mermilliod, 1991) and the Andruk catalogue of 1141 UBVR stellar magnitudes (Andruk at al.,1995). All original coordinates have the different epoch and equinox. We performed the cross reference of stars from these three catalogues with the Tycho2, UCAC4 and XPM catalogues and created a new photometric catalogue on the epoch and equinox of J2000.0.
\end{abstract}

Keywords: Photometric - methods: data analysis - catalogues virtual observatory tools - astrometry - techniques

\section{Introduction}

For the goals and objectives of photometric calibration of the astronomical photographic plates exposed for the sky areas from $-30^{\circ}$ to $90^{\circ}$ was created the first version of the compiled star catalogue of the positions, proper motions and photoelectric U(W)BVR stellar magnitudes in the Johnson system. The reason for the creation of this catalogue was the low precision of the equatorial coordinates and the absence of any information about the proper motions of stars, that have photoelectric U(W)BVR stellar magnitudes in all source catalogues. For our purposes were chosen three source catalogues:

1. Photometric catalogue of UBV stellar magnitudes (Kornilov et al., 1991)

2. Photometric catalogue of WBVR stellar magnitudes (Mermilliod et al., 1991)

3. Photometric catalogue of UBVR stellar magnitudes of the MEGA project (Andruk et al., 1995; Andruk 1996; Andruk 1996; Andruk 1996).

The corresponding number of stars with the photoelectric stellar magnitudes in three source catalogues is following: 13586 (down to $\delta \leq-26^{\circ}$ ), 47022 (down to $\delta \leq-30^{\circ}$ ) and 1141 (down to $\delta \leq-13^{\circ}$ ). Altogether the whole list has 61749 stars. The precision of the equatorial coordinates of the stars in these catalogues is not high enough, (especially for the second catalogue), that complicated the identification of the calculated coordinates of stars obtained as the result of the processing of digitized images of photographic plates. The difficulties by the identification are significant for the faint stars and growing up for the photographic plates recorded in the first half of the twentieth century.

\section{The results of the identification with the Tycho2, UCAC4 and XPM catalogues}

All stars of the created list were consistently identified with the stars of the Tycho2, UCAC4 (Zacharias et al., 2013) and XPM (Fedorov P. et al., 2009) catalogues. The corresponding amounts of stars of the individual source catalogues identified with the Tycho 2 catalogue were following: the 38837, 13202 and 633 stars for the first, second and third catalogues respectively. Then the not identified stars from the whole list were compared with the UCAC4 catalogue. The results of this second identification were: the 7803, 289 and 460 stars for the first, second and third source catalogues respectively. The same procedure of the identification for the rest of not identified stars using the XPM catalogue gave out the following results: the 75, 3 and 7 stars. So the first version of our catalogue contain 61309 stars. Due to the errors of identification and because of the duality of a certain amount of stars, the number of the starts in the final catalogue will be decreased.

3. The using of the two exposures on the same photographic plate for the photometry

The combination of characteristic curves of the two exposures on the same photographic plate makes possible to build a new combined characteristic curve for the whole interval of stellar magnitudes on the photographic plate. The photoelectric stellar magnitudes (Bpe) from the source catalogues were used as the photoelectric standards. The steps and methods of the construction of such combined characteristic curves for photographic plates, are shown on the figures 1 and 2 . The photometric field errors were also taken into account. The difference between stellar magnitudes of two exposures in the scale of the photoelectric standards (Bpe) is $\Delta \mathrm{B}=3.223 \mathrm{mag}$. The errors of photome- 
try are $\sigma 1= \pm 0.229$ mag for the first exposure and $\sigma 2= \pm$ $0.374 \mathrm{mag}$ for the second one. The characteristic curves for the binding of the instrumental photometric values of $\mathrm{ml}$ and $\mathrm{m} 2$ with the scale of photoelectric standards Bpe are displayed on the d-panel. The photometric errors are $\sigma 1= \pm$ $0.146 \mathrm{mag}$ and $\sigma 1= \pm 0.179 \mathrm{mag}$.
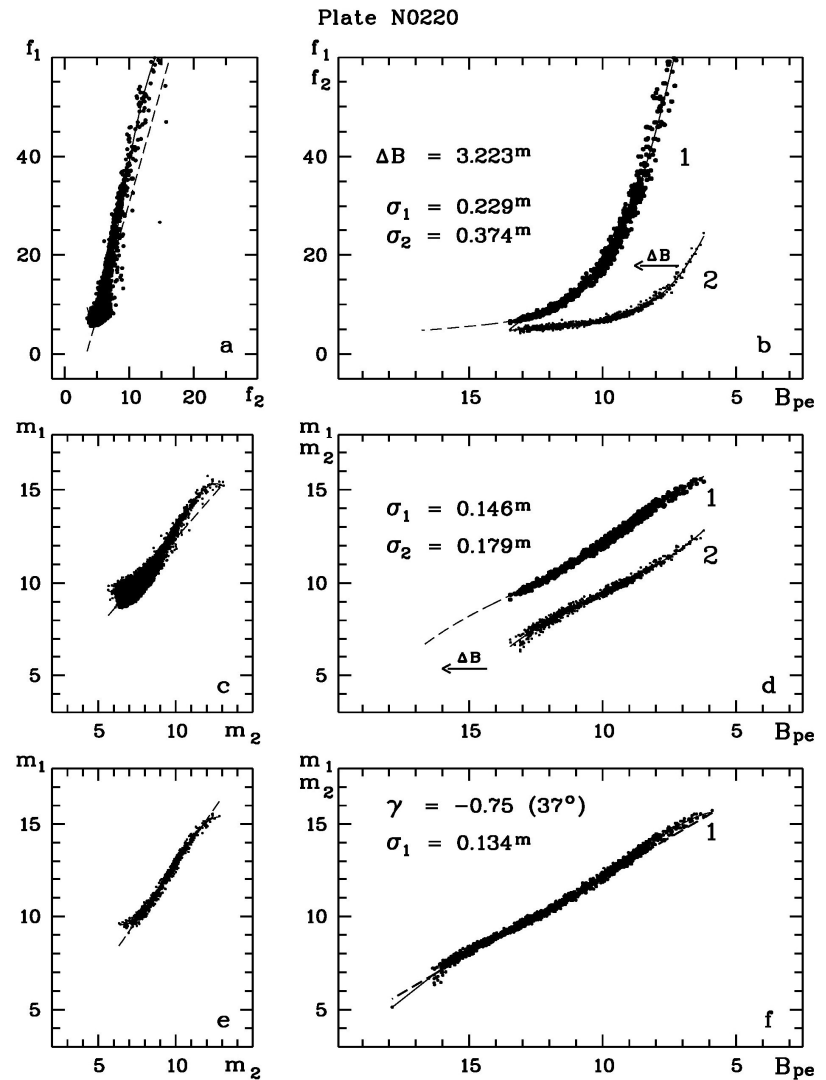

Figure 1: Photometry of stars using the information about the two exposures for the photographic plate N0220.
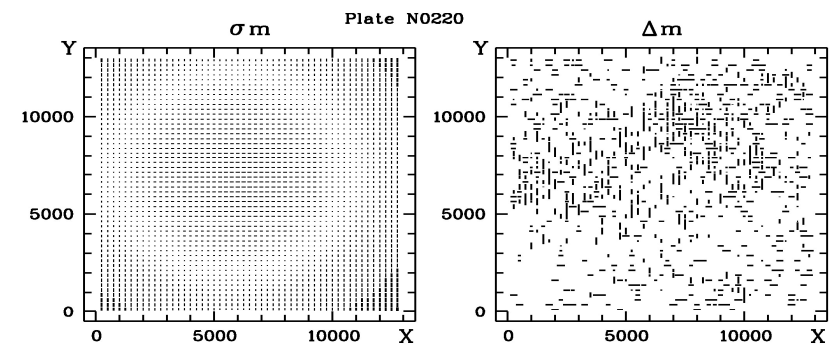

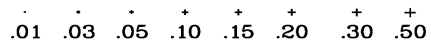

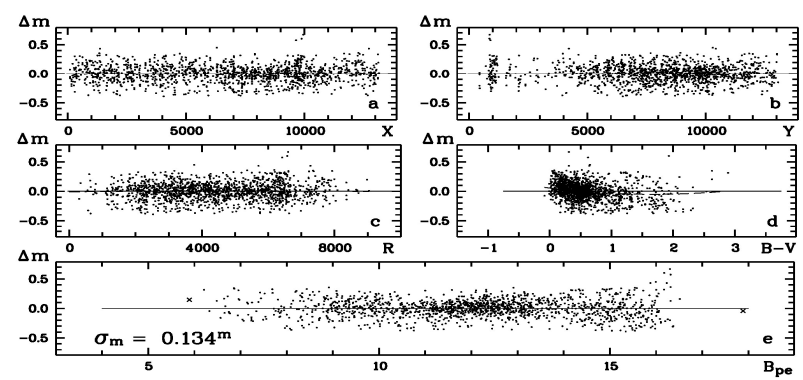

Figure 2: The photometric field errors (upper panels and panels a, b, c, d) for the photographic plate N0220.

\section{About the compiled catalogue of photoelectric U(W)BVR magnitudes}

As a result of this succeeding identification was created a compiled star catalogue of the equatorial coordinates and proper motions in the system of the Tycho 2 catalogue and photoelectric U(W)BVR magnitudes adopted to the Johnson system. The electronic form of this catalogue is presented in the 14 columns. The first seven columns contain information from the original source catalogues: equatorial coordinates $\alpha, \delta$ ( 1 and 2 columns); U(W), B, V and $\mathrm{R}$ magnitudes $(3,4,5$ and 6 columns); the 7 column contains the references on the original photometric catalogues (1 - Andruk et al., 1995, 2 - Kornilov et al., 1991 and 3 - Mermilliod et al., 1991). The next seven columns contain the results of the identification: the equatorial coordinates of the stars on the epoch and equinox $2000.0(8$ and 9 columns); the proper motions $\mu \alpha \operatorname{Cos} \delta$ and $\mu \delta$ (arcsec/year, 10 and 11 columns); B and V magnitudes from the Tycho2, UCAC4 and XPM catalogues recorded in columns 12 and 13; the last column (14) contains the references on the catalogues where data taken from (1 Tycho2, 2 - UCAC4 and 3 - XPM).

\section{Conclusion}

This work was performed within the framework of work on the creation of software for data reduction of Joint Digital Archive of Ukrainian Virtual Observatory (Vavilova et al., 2012; Vavilova et al., 2012). The photoelectric calibration of the photographic plates was already used by the authors in series of works (Andruk et al., 2010, Andruk et al., 2014, Andruk et al., 2016, Kazantseva et al., 2014, Protsyuk et al., 2014). The created catalogue will be posted on the websites of the RIO NAO (Ukraine), MAO NASU (Ukraine) observatories and as well as will be transferred to the Strasbourg astronomical Data Centre.

Acknowledgements. The authors are thankful to anybody who has read this contribution to the end.

\section{References}

Andruk V.M. et al.: 1995, Astron. Nachr., 316, N4, 225. Andruk V.M. et al.: 1996, Astron. Nachr., 317, N2, 49. Andruk V.M. et al.: 1996, Astron. Nachr., 317, N2, 127. Andruk V.N.: 1996, Kinem. Phys. Cel. Bodies, 12, N4, 60. Andruk V.M. et al.: 2010, Kinem. Phys. Cel. Bodies, 26, N3, 146.

Andruk V.M. et al.: 2014, Odessa Astron. Publ., 27, N1, 53. Andruk V.M. et al.: 2016, Kinem. Phys. Cel. Bodies, 32, N1, 56.

Fedorov P. et al.: 2009, Mon. Not. R. Astron. Soc., 393, 133.

Kazantseva L.V. et al.: 2015, Kinem. Phys. Cel. Bodies, 31, N1, 58.

Kornilov V.G. et al.: 1991, Trudy GAIS, 63, 1.

Mermilliod J.C.: 1991, Homogeneous means in the UBV system.

Protsyuk Yu.I. et al.: 2014, Kinem. Phys. Cel. Bodies, 30, N6, 54.

Vavilova I.B. et al.: 2012, Kinem. Phys. Cel. Bodies, 28, N2, 85.

Vavilova I.B. et al.: 2012, Baltic Ast., 21, N3, 356.

Zacharias N. et al.: 2013, Astron. J., 145. 44. 